to para uma diferença mínima significativa de $10 \%$ e de 8 mudas por tratamento quando a diferença passa para $20 \%$.

Palavras-chave: heterogeneidade, precisão, delineamento.

Three experiments were conducted to determine the optimum experimental plot size and shape of Eucalyptus saligna Smith. The experiments were established in Barda Negra Garden Market, in Barra do Ribeiro - RS. Plants were distributed in a $3 \times 2 \mathrm{~m}$ arrangement. Plants were evaluated with three different ages: 5.5 years, 15 months and during the nursing stage. Seven different analysis methods were used to evaluate the optimum experimental plot size and shape based only on data collected from experi- ments with 5.5 years old and 15 months old. The nursery experiments were used to determine the sample frequency for minimum statistical difference of 5, 10 and $20 \%$. It was observed statistical difference among the sevem methods used for comparisons. The optimum plot size ranged from 2 to 26 basic units for plants with 5.5 years old and, from one to $16^{\circ}$ basic units for plants with 15 months old. Results also indicated that the optimum plot size could be 16 and 8 basic units for plants with 5.5 years old and 15 months old, respectively. Reducing the plot size and increasing the repetition number were more adequate to analyze this type of experiments. Nursery experiments should be conducted in a completely randomized block design using at least 26 seedling for each treatment.

Key words: heterogeneity, precision, design.

\title{
PARÂMETROS DA PRECISÃO EXPERIMENTAL DAS PRINCIPAIS CULTURAS ANUAIS DO ESTADO DO RIO GRANDE DO SUL ${ }^{1}$
}

\author{
EXPERIMENTAL PRECISION PARAMETERS FOR MAIN ANNUAL \\ CROPS OF RIO GRANDE DO SUL STATE
}

\author{
Autor: Alessandro Dal'Col Lúcioº \\ Comissão de Avaliação: Lindolfo Storck ${ }^{3}$ \\ Arno Bernardo Heldwein ${ }^{4}$ \\ Sidinei José Lopes 5
}

Na experimentação agronômica, a qualidade de um determinado ensaio é um dos fatores que indica a confiabilidade dos resultados obtidos. Esta qualidade pode ser descrita através das estatísticas: coeficiente de variação (CV), coeficiente de precisão
(CP) e diferença mínima significativa (DMS). Este estudo teve por objetivo, identificar a distribuição teórica das estatísticas CV, CP e DMS, para estabelecer limites de precisão dos ensaios para as culturas do milho, arroz, soja e trigo, o controle de qualidade dos

\footnotetext{
${ }^{1}$ Dissertação de Mestrado apresentada pelo primeiro autor, em 10.01.97, ao Curso de Pós-graduação em Agronomia, Centro de Ciências Rurais (CCR), Universidade Federal de Santa Maria (UFSM), para a obtenção do grau de Mestre em Agronomia, área de Produção Vegetal.

${ }^{2}$ Engenheiro Agrônomo, Mestre em Agronomia, Produção Vegetal, UFSM.

${ }^{3}$ Engenheiro Agrônomo, Dr., Professor Titular, Departamento de Fitotecnia, CCR, UFSM, 97119-900 Santa Maria, RS. Bolsista do CNPq. Autor para correspondência.

${ }^{4}$ Engenheiro Agrônomo, Dr, Professor Adjunto, Departamento de Fitotecnia, CCR, UFSM.

${ }^{5}$ Engenheiro Agrônomo, Mestre, Prof. Ass. do Dep. de Fitotecnia, CCR, UFSM Recebido para publicaçion em 18.03.97. A provado em 02.04.97.
} 
ensaios e, identificar algumas técnicas de manejo que possam afetar esta qualidade. Fez-se um estudo de 1655 ensaios de competição de cultivares, sendo destes, 549 com a cultura do milho, 104 com a cultura do arroz, 480 com a cultura da soja e 522 com a cultura do trigo. A hipótese de aderência dos valores dos CV, CP e DMS à distribuição normal foi rejeitada em nível de $1 \%$ de significância para todas as culturas avaliadas, mas para subgrupos de ensaios com manejos culturais semelhantes, dentro de cada cultura, a normalidade dos valores das estatísticas foi mais evidente. Em ensaios com a cultura do milho, manejos como desbaste aumenta a precisão experimental e a aplicação de inseticidas reduz esta precisão. $O$ controle de ervas daninhas em soja, aumenta a precisão nos ensaios. A pré-germinação em arroz e o tratamento de sementes em soja e trigo são capazes de aumentar esta precisão. Em ensaios com a cultura da soja, o uso de adubação, seja no plantio ou em cobertura, reduz a precisão experimental, enquanto que o controle de ervas daninhas, o tratamento de sementes e a aplicação de inseticidas aumenta esta precisão. Em ensaios com a cultura do trigo, o uso do tratamento de sementes e aplicação de inseticidas, aumenta-se a precisão. Para o controle de qualidade dos ensaios de competição de cultivares de milho, arroz, soja e trigo, foi determinado limites de classe para serem usados como parâmetros na classificação das estatísticas CV, CP e DMS, sendo que estas são equivalentes entre si, quando se trata da medida da precisão experimental, sendo recomendado o uso da estatística DMS, ao invés das outras, devido sua fácil estimativa e porque mostra a importância econômica.

Palavras-chave: delineamento, qualidade, precisão experimental.
In agricultural research the experimental quality is a factor that indicates reliable results. This quality may be described by variation (CV) and precision (CP) coeficients and least significant difference (LSD) which also are used to measure experimental precision. The study main goal was to identify the theoretical distribution of statistical parameters to establish precision limits for maize, rice, soybean and wheat crops. Another objetive was to established parameters for experimental quality standards and identify management technics which affect their quality. It was studied 1655 experiments with cultivars: 549 of maize, 104 of rice, 480 of soybeans and 522 of wheat. The adherence hypothesis for CV, CP and LSD to normal distribution was rejected ( $1 \%$ level) for all studied crops. However the normaly of statistical parameters were more evident for sub-groups with same crop management and for each crop. Crop management such as plant population control, herbicide and insecticide application reduce precision for maize experiments. Herbicide application, start or band fertilization reduced the precision for rice experiments while pre-germination increase experimental precision. Start and band fertilization for soybean experiments reduced precision while herbicide and insecticide application and seed treatments increased experimental precision. The precision was increased for wheat crop experiments was increased when seed was treated and insecticides were applied. It was established limits for CV, CP and LSD to be used in experimental quality control. The later parameters were equivalent among then to measure experimental precision. However, it was recomended to use the LSD due to the facility of estimation and the economical importance.

Key words: design, quality, experimental precision. 